Paul Joseph Thuluvath, Professor, Series Editor

\title{
Hepatorenal syndrome
}

\author{
Sharon Turban, Paul J Thuluvath, Mohamed G Atta
}

Sharon Turban, Mohamed G Atta, Department of Medicine, Division of Nephrology, Johns Hopkins School of Medicine and the Johns Hopkins Hospital, Baltimore, Maryland, United States Paul J Thuluvath, Department of Medicine, Division of Gastroenterology, Johns Hopkins School of Medicine and the Johns Hopkins Hospital, Baltimore, Maryland, United States Correspondence to: Mohamed G Atta, MD, MPH, Johns Hopkins University, Division of Nephrology, 1830 East Monument Street, Suite 416, Baltimore, Maryland-21205, United States.matta1@jhmi.edu

Telephone: +1-410-9555268 Fax: +1-410-9550485

Received: 2007-01-11 Accepted: 2007-03-08

\begin{abstract}
Hepatorenal syndrome (HRS) is a "functional" and reversible form of renal failure that occurs in patients with advanced chronic liver disease. The distinctive hallmark feature of HRS is the intense renal vasoconstriction caused by interactions between systemic and portal hemodynamics. This results in activation of vasoconstrictors and suppression of vasodilators in the renal circulation. Epidemiology, pathophysiology, as well as current and emerging therapies of HRS are discussed in this review.
\end{abstract}

(c) 2007 WJ G. All rights reserved.

Key words: Acute renal failure; End stage liver disease; Hepatorenal syndrome; Transjugular intrahepatic portosystemic shunts; Dialysis; Liver transplantation

Turban S, Thuluvath PJ, Atta MG. Hepatorenal syndrome. World J Gastroenterol 2007; 13(30): 4046-4055

http://www.wjgnet.com/1007-9327/13/4046.asp

\section{INTRODUCTION}

The association between liver disease and renal dysfunction was reported more than a century ago when patients with chronic liver disease and normal renal histology were found to develop oliguric renal failure (Flint A, Am J Med Sci 1863). This led to proposed links between renal dysfunction and the derangement in systemic circulation secondary to the liver failure ${ }^{[1]}$.

Renal failure in patients with liver disease may be caused by several factors, including shock, sepsis, nephrotoxic medications, intrinsic renal diseases, or volume depletion secondary to diuresis or large-volume paracentesis. However, renal failure may also occur in patients with liver disease in the absence of the above factors and in the absence of major renal histological changes. This is referred to as hepatorenal syndrome (HRS). HRS is considered a "functional" and reversible form of renal failure $^{[2-6]}$. The International Ascites Club defined HRS as: "a syndrome that occurs in patients with advanced chronic liver disease and advanced hepatic failure and portal hypertension characterized by impaired renal function and marked abnormalities in the arterial circulation and activity of the endogenous vasoactive systems. In the kidney, there is marked renal vasoconstriction that results in low glomerular filtration rate (GFR). In the extrarenal circulation, there is a predominance of arterial vasodilation, that results in reduction of total systemic vascular resistance and arterial hypotension". The incidence of HRS in patients with chronic liver disease is not well studied. In one study of 234 non-azotemic patients with liver disease who had ascites and cirrhosis, $18 \%$ developed HRS at 1 year, and $39 \%$ by 5 years ${ }^{[7]}$. Although HRS usually occurs in patients with advanced cirrhosis, it has also been described in patients without ascites in the setting of acute fulminant hepatic failure ${ }^{[8]}$.

\section{PATHOPHYSIOLOGY}

Approximately $80 \%$ of hospitalized patients with cirrhosis and ascites have decreased renal perfusion due to moderate vasoconstriction in the renal circulation, which predisposes them to develop $\mathrm{HRS}^{[7-9]}$. In 10\%-17\% of these patients, renal vasoconstriction becomes intense enough to cause significant renal hypoperfusion, resulting in $\mathrm{HRS}^{[7,10]}$. This intense renal vasoconstriction is the distinctive hallmark feature of $\mathrm{HRS}^{[11,12]}$. The mechanisms of renal vasoconstriction are complex and multifactorial, and are incompletely understood. There appear to be interactions between changes in systemic hemodynamics, portal hypertension, activation of vasoconstrictors, and suppression of vasodilators in the renal circulation ${ }^{[13,14]}$. In contrast, significant vasodilation occurs in the splanchnic arterial bed secondary to increased production of local vasodilators, predominantly nitric oxide ${ }^{[15]}$. Other vasodilators hypothesized to play a role in splanchnic arterial vasodilation include prostacyclin, prostaglandin E2, atrial natriuretic peptide, kallikreins, and kinins ${ }^{10,16,17]}$. This splanchnic vasodilation is believed to lead to compensatory responses by activating vasoconstrictors including the renin- 


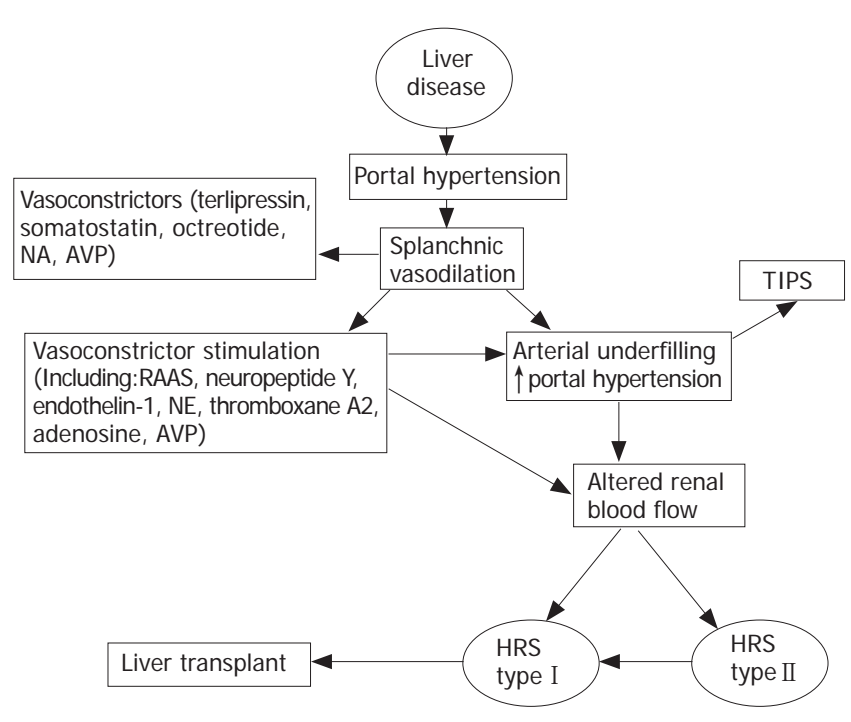

Figure 1 Pathophysiology of HRS and potential therapeutic interventions. NA: noradrenalin; AVP: arginine vasopressin; RAAS: renin-angiotensin system; NE: norepinephrine; TIPS: transjugular intrahepatic portosystemic shunt.

angiotensin-aldosterone system (RAAS), neuropeptide Y, endothelin-1, norepinephrine, thromboxane A2, adenosine, and antinatriuretic agents such as arginine vasopressin (AVP). This leads to retention of sodium and water in addition to renal vasoconstriction ${ }^{[15,18,19]}$. Other factors such as the absence of or decrease in glomerulopressin or other liver-borne diuretic factors (factors that are released by the liver and target the kidney) could also contribute to renal failure $^{[20]}$. In recent years, the potential role of cirrhotic cardiomyopathy has been postulated in the pathogenesis of HRS. Ruiz-del-Arbol et al have demonstrated that HRS is due to decreased cardiac output in the setting of a severe arterial vasodilation ${ }^{[21]}$. Similar circulatory events were also shown in cirrhotic patients who developed spontaneous bacterial peritonitis ${ }^{[22]}$.

In the early stages of cirrhosis, the activation of local vasodilators may overcome the renal vascular effects of systemic vasoconstrictors, maintaining adequate renal perfusion ${ }^{[23]}$. As liver disease progresses, the renal vasodilators are no longer able to antagonize the circulating vasoconstrictors, and this results in severe renal vasoconstriction and impaired renal blood flow. In addition, the hypoperfusion itself may lead to further intrarenal vasoconstriction. Figure 1 summarizes the complex pathways involved in the development of HRS and potential therapeutic interventions.

\section{CLASSIFICATION OF HRS}

There are two types of HRS (Table 1). Type 1 is characterized by a rapid elevation in blood urea nitrogen (BUN) and creatinine, often defined as a 100\% increase in serum creatinine, reaching a level higher than $2.5 \mathrm{mg} / \mathrm{dL}$ in less than two weeks ${ }^{[2]}$. The mortality of patients with type 1 HRS has been reported to be $80 \%$ at two weeks ${ }^{[7]}$. Type 2 HRS, on the other hand, generally follows a slower course and has a better prognosis. It is usually characterized by recurrent, diuretic-resistant ascites ${ }^{[10,14]}$, and is thought to be due to significant activation of anti-natriuretic systems ${ }^{[2]}$.
Table 1 Clinical types of HRS

Type 1

(1) $100 \%$ increase in serum creatinine to a level higher than $2.5 \mathrm{mg} / \mathrm{dL}$ or a $50 \%$ reduction of the initial 24 -h creatinine clearance to a level lower than $20 \mathrm{~mL} / \mathrm{min}$ in less than $2 \mathrm{wk}$

(2) Very poor short-term outcome

Type 2

(1) Serum creatinine $>1.5 \mathrm{mg} / \mathrm{dL}$, without meeting the criteria for type $1 \mathrm{HRS}$

(2) Refractory ascites is usually present

(3) Prognosis is not as poor as with type 1

\section{PRECIPITATING FACTORS}

HRS may develop spontaneously without known precipitating factors, but there are known triggers ${ }^{[14,24]}$. Spontaneous bacterial peritonitis (SBP) has been associated with type $1 \mathrm{HRS}$ in approximately $20 \%$ of cases $^{[21,25]}$, even with treatment and resolution of the infection. These patients have a very poor outcome. HRS may also occur after therapeutic paracentesis without plasma expansion ${ }^{[26,27]}$. Gastrointestinal bleeding has also been identified as a precipitant of HRS, but this usually occurs in patients with hypovolemic shock. In this setting, acute renal tubular ischemic injury or necrosis is more likely to be the cause of acute renal failure than $\mathrm{HRS}^{[28]}$. There is no clear evidence to support diuretic-induced volume depletion as a precipitating factor of $\mathrm{HRS}^{[14]}$. Other factors that have been associated with an increased risk of developing HRS in patients with ascites and cirrhosis include severe urinary sodium retention, spontaneous dilutional hyponatremia, and a mean arterial blood pressure less than $80 \mathrm{mmHg}$. There is not a direct linear association between the severity of liver failure and the incidence of HRS, but HRS is usually seen in patients with advanced liver disease and portal hypertension ${ }^{[14,23]}$.

\section{DIAGNOSIS OF HRS}

There are no specific clinical or laboratory findings for the diagnosis of HRS. The diagnosis is established based on predefined criteria in the appropriate clinical setting (Table 2). Patients with advanced liver disease may develop renal failure from a number of causes other than HRS, and these causes must be excluded before making a diagnosis of HRS. Common causes of renal failure in patients with cirrhosis include volume depletion (which could be secondary to over-diuresis, diarrhea, or poor fluid intake), nephrotoxic medications (commonly non-steroidal anti-inflammatory agents and aminoglycosides), allergic interstitial nephritis, acute tubular necrosis (from various factors including shock), contrast nephropathy, and intrinsic renal diseases such as glomerulonephritis. A renal biopsy may rarely be necessary if the diagnosis is unclear, mainly to exclude other treatable renal diseases. It is also important to note that there are significant limitations in using serum creatinine as a marker of renal function in patients with liver disease. Patients with advanced liver disease usually have reduced muscle mass and hence low endogenous production of creatinine. When creatinine clearances in cirrhotic patients were compared with inulin clearances, the 
glomerular filtration rates were significantly overestimated ${ }^{[29]}$. Alternative diagnostic approaches have been applied in order to overcome the limitation of serum creatinine values in this population. Platt $e t a l^{[0]}$ examined the utility of Doppler ultrasonography to assess the resistive indices of the renal vasculature. In their study of 180 patients with liver disease without azotemia, $42 \%$ of the patients were found to have an increase in renal vascular resistive indices. Of those patients, $55 \%$ subsequently developed renal failure as compared to $6 \%$ of those with normal resistive indices. The sensitivity and specificity of the resistive index in detecting renal failure were estimated at $71 \%$ and $80 \%$ respectively in a group of cirrhotic patients ${ }^{[30]}$. However, this technique is operator-dependent and is still under investigation, and therefore is not currently recommended as a standard method to diagnose HRS.

\section{PROGNOSIS}

The prognosis of HRS is extremely poor. The median survival time of patients with type 1 HRS is less than 2 wk, with less than $10 \%$ surviving their hospital stay ${ }^{[7]}$. The survival time of patients with type $2 \mathrm{HRS}$, although still short, is significantly longer, with a median survival time of approximately $6 \mathrm{mo}^{[14]}$.

\section{MANAGEIMENT}

\section{Prevention of HRS}

Prevention of HRS is potentially possible in some high-risk patients. In patients with SBP, administering intravenous albumin $(1.5 \mathrm{~g} / \mathrm{kg}$ upon diagnosis, and then $1 \mathrm{~g} / \mathrm{kg}$ after $48 \mathrm{~h}$ ) in addition to antibiotics has been shown to decrease the incidence of HRS and to decrease hospital mortality as compared with treatment with antibiotics alone ${ }^{[26]}$. The authors of that study postulated that the administration of albumin prevented circulatory dysfunction by maintaining effective arterial blood volume and therefore prevented vasoconstrictor activation. However, albumin is expensive, and more studies are needed to determine if lower doses of albumin or less expensive artificial plasma expanders are as effective. In one study, administration of pentoxifylline (400 $\mathrm{mg}$ orally three times a day) to patients with severe acute alcoholic hepatitis decreased the incidence of HRS as well as the short-term mortality rate compared to placebo ${ }^{[31]}$. This benefit may be related to the inhibition of tumor necrosis factor production. Although both of the above studies support the idea of preventing renal failure in the setting of liver failure, there are no data evaluating the long-term survival benefit in this population. Moreover, there have been no further confirmatory studies.

\section{General management measures}

In patients with type $1 \mathrm{HRS}$, diagnostic paracentesis is generally recommended to evaluate for SBP. In addition, diuretics should be discontinued as they may potentially worsen renal function. In the absence of contraindications, patients with type 1 HRS should also be evaluated for expedited liver transplantation.
Table 2 Diagnostic criteria of hepatorenal syndrome

Major criteria (all must be present for the diagnosis of HRS)

(1) Advanced hepatic failure (acute or chronic liver disease) and portal hypertension

(2) Low GFR defined as serum creatinine $>1.5 \mathrm{mg} / \mathrm{dL}$ or creatinine clearance $<40 \mathrm{~mL} / \mathrm{min}$

(3) Absence of shock, significant volume losses, ongoing infection, or treatment with nephrotoxic medications

(4) Absence of a sustained improvement in renal function after cessation of diuretics and expansion of plasma volume with $1.5 \mathrm{~L}$ of isotonic fluids

(5) Urine protein excretion $<500 \mathrm{mg} / \mathrm{dL}$ with no ultrasonographic evidence of obstruction or parenchymal renal disease

Additional criteria (not necessary for the diagnosis, but provide supportive evidence)

(1) Urine volume $<500 \mathrm{~mL} / \mathrm{d}$

(2) Urine sodium $<10 \mathrm{mEq} / \mathrm{L}$

(3) Urine osmolality greater than plasma osmolality

(4) Urine red blood cells $<50$ per high-power field

(5) Serum sodium concentration $<130 \mathrm{mEq} / \mathrm{L}$

${ }^{1}$ Adapted from Arroyo et $a l^{[2]}$.

\section{Pharmacological therapy}

Several systemic vasoconstrictors have been utilized in the treatment of type 1 HRS as summarized in Table 3. Renal vasodilators such as dopamine and prostaglandin analogues are no longer recommended due to their side effect profile and the lack of clinical evidence to support their use. Other potential forms of therapy that have not been extensively tested include endothelin blockers ${ }^{[32]}$ and $\mathrm{N}$-acetylcysteine $\mathrm{e}^{[33]}$.

The rationale behind the use of vasoconstrictors along with plasma expansion is that they will counteract the splanchnic arterial vasodilation, which is hypothesized to be the initial event in the pathogenesis of HRS. Unopposed splanchnic arterial vasodilation may cause a decrease in effective arterial volume which in turn triggers the activation of vasoconstrictors ${ }^{[2,34]}$. Vasoconstrictors that have been widely used for type 1 HRS include vasopressin analogues (ornipressin and terlipressin), a somatostatin analogue (octreotide), and alpha-adrenergic analogues (midodrine and noradrenalin). In most studies, albumin was administered concurrently.

The vasopressin analogues are effective in causing marked splanchnic vasoconstriction. Ornipressin, although effective in treating HRS, may cause significant ischemic side effects and is not currently recommended for the management of $\mathrm{HRS}^{[35]}$. Studies using terlipressin, the long-acting analogue of vasopressin, have shown significant improvement in renal function in approximately $60 \%-75 \%$ of patients, with a lower than $5 \%$ incidence of ischemic side effects ${ }^{[36-43]}$. In these studies, patients with Child-Pugh scores less than or equal to 13 and/or those who received albumin infusions had a more favorable outcome. However, it is important to note that GFR was not normalized in most patients who responded ${ }^{[37,39]}$. Approximately $15 \%$ of patients had recurrence of HRS once treatment was discontinued. Small, short-term, non-randomized studies suggest that treatment with terlipressin may also improve renal function in patients with type $2 \mathrm{HRS}^{[34]}$. Terlipressin is not currently licensed 
Table 3 Non-invasive therapies

\begin{tabular}{|c|c|c|c|c|c|c|c|c|}
\hline $\begin{array}{l}\text { Author } \\
\text { and Year }\end{array}$ & $\begin{array}{l}n \text { (\# Type } 1 \text {, } \\
\text { \# Type 2) }\end{array}$ & Study design & Intervention & $\begin{array}{l}\text { Outcome } \\
\text { measures }\end{array}$ & $\begin{array}{l}\text { Mean } \\
\text { baseline } \\
\text { SCr }\end{array}$ & $\begin{array}{l}\text { Mean } \\
\text { follow-up } \\
\text { SCr }\end{array}$ & Other results & Comments \\
\hline $\begin{array}{l}\text { Moreau et al } \\
2002^{[38]}\end{array}$ & $99(99 / 0)$ & $\begin{array}{l}\text { Multicenter, } \\
\text { retrospective }\end{array}$ & $\begin{array}{l}\text { Terlipressin } \\
\text { (75\% received } \\
\text { albumin) }\end{array}$ & $\begin{array}{l}\text { Reduction of } \\
\text { SCr to } \\
<130 \mu \mathrm{mol} / \mathrm{L} \\
\text { or a decrease of } \\
\text { at least } 20 \% \text { at } \\
\text { end of treatment) }\end{array}$ & $\begin{array}{l}272 \pm 114 \\
\mu \mathrm{mol} / \mathrm{L}\end{array}$ & $\begin{array}{l}\text { Responders: } \\
138 \pm 59 \\
\mu \mathrm{mol} / \mathrm{L} \\
\text { Nonresponders: } \\
382 \pm 210 \\
\mu \mathrm{mol} / \mathrm{L}\end{array}$ & $\begin{array}{l}\text { Renal function } \\
\text { improved in } \\
58 \% \text { of } \\
\text { patients. }\end{array}$ & $\begin{array}{l}\text { Twenty-three patients } \\
\text { had adverse events } \\
\text { that may have been } \\
\text { terlipressin-related. } \\
\text { Three patients } \\
\text { required RRT } 40 \% \\
\text { survival at } 1 \text { mo. }\end{array}$ \\
\hline $\begin{array}{l}\text { Kiser et al } \\
2005^{[44]}\end{array}$ & $43(32 / 11)$ & $\begin{array}{l}\text { Observational } \\
\text { (retrospective } \\
\text { cohort) }\end{array}$ & $\begin{array}{l}\text { Vasopressin } \\
(\mathrm{AVP}) \text { vs } \\
\text { octreotide vs } \\
\text { combination }\end{array}$ & $\begin{array}{l}\text { Clinical } \\
\text { response; } \\
\mathrm{SCr} 1.5 \mathrm{mg} / \mathrm{dL} \\
\text { or less }\end{array}$ & $\begin{array}{l}3.9 \pm 3.3 \\
\mathrm{mg} / \mathrm{dL}\end{array}$ & $\begin{array}{l}\text { Responders: } \\
\text { SCr decreased } \\
\text { by } 62 \% \pm 9 \% \\
\text { Nonresponders: } \\
\text { SCr increased } \\
\text { by } 46 \% \pm 119 \%\end{array}$ & $\begin{array}{l}42 \% \text { complete } \\
\text { response with } \\
\text { AVP vs } 38 \% \\
\text { with AVP } \\
\text { and octreotide } \\
\text { vs } 0 \% \text { with } \\
\text { octreotide alone. }\end{array}$ & $\begin{array}{l}\text { No adverse effects } \\
\text { related to AVP. } \\
\text { RRT rates: } 50 \% \text { in } \\
\text { AVP group, } 58 \% \text { in } \\
\text { combination group, } \\
\text { and } 31 \% \text { in octreotide } \\
\text { alone group. }\end{array}$ \\
\hline $\begin{array}{l}\text { Solanki et al } \\
2003^{[43]}\end{array}$ & $24(24 / 0)$ & $\begin{array}{l}\text { Randomized } \\
\text { placebo- } \\
\text { controlled } \\
\text { single-blind }\end{array}$ & $\begin{array}{l}\text { Terlipressin } \\
\text { vs placebo (all } \\
\text { patients received } \\
\text { albumin) for } \\
4-15 \mathrm{~d}\end{array}$ & $\begin{array}{l}\text { Reversal of } \\
\text { HRS and } \\
\text { survival at } \\
15 \mathrm{~d}\end{array}$ & $\begin{array}{l}\text { Terlipressin: } \\
2.9 \pm 0.1 \\
\mathrm{mg} / \mathrm{dL} \\
\text { Placebo: } \\
2.2 \pm 0.2 \\
\mathrm{mg} / \mathrm{dL}\end{array}$ & $\begin{array}{l}\text { Terlipressin: } \\
1.2 \pm 0.2 \mathrm{mg} / \mathrm{dL} \\
\text { at d } 15 \text { Placebo: } \\
\text { no survival at } \mathrm{d} \\
15(\mathrm{SCr} 3.9 \pm 0.2 \\
\mathrm{mg} / \mathrm{dL} \text { on } \mathrm{d} 8)\end{array}$ & $\begin{array}{l}\text { In terlipressin } \\
\text { group, } 5 \text { of } 12 \\
\text { patients survived. } \\
\text { None survived } \\
\text { by d } 15 \text { in } \\
\text { placebo group. }\end{array}$ & \\
\hline $\begin{array}{l}\text { Ortega et al } \\
2002^{[39]}\end{array}$ & $21(16 / 5)$ & $\begin{array}{l}\text { Prospective, } \\
\text { nonrandomized }\end{array}$ & $\begin{array}{l}\text { Terlipressin with } \\
\text { albumin } v \text { s } \\
\text { without albumin } \\
\text { for } 4-14 \mathrm{~d}\end{array}$ & $\begin{array}{l}\mathrm{SCr} \\
1.5 \mathrm{mg} / \mathrm{dL} \\
\text { or lower }\end{array}$ & $\begin{array}{l}\text { Terlipressin } \\
\text { with albumin: } \\
3.6 \pm 1.5 \\
\mathrm{mg} / \mathrm{dL} \\
\text { Terlipressin } \\
\text { without } \\
\text { albumin: } \\
3.4 \pm 0.3 \\
\mathrm{mg} / \mathrm{dL}\end{array}$ & $\begin{array}{l}\text { Terlipressin } \\
\text { with albumin: } \\
1.5 \pm 0.2 \mathrm{mg} / \mathrm{dL} \\
\text { Terlipressin } \\
\text { without } \\
\text { albumin: } \\
3.4 \pm 0.7 \mathrm{mg} / \mathrm{dL}\end{array}$ & $\begin{array}{l}10 \text { of } 13 \text { patients } \\
\text { who received } \\
\text { terlipressin } \\
\text { and albumin } \\
\text { responded. } \\
\text { Of } 8 \text { patients } \\
\text { who received } \\
\text { terlipressin alone, } \\
2 \text { responded. }\end{array}$ & $\begin{array}{l}\text { One patient had } \\
\text { ischemic side } \\
\text { effects } \\
\text { (finger ischemia). } \\
\text { At } 1 \text { mo, there } \\
\text { was a } 5 \% \\
\text { recurrence of } \\
\text { HRS after } \\
\text { complete response. }\end{array}$ \\
\hline $\begin{array}{l}\text { Pomier- } \\
\text { Layrargues } \\
\text { et al } 2003^{[61]}\end{array}$ & 19 (NS) & $\begin{array}{l}\text { Randomized, } \\
\text { double-blind, } \\
\text { placebo- } \\
\text { controlled, } \\
\text { crossover }\end{array}$ & $\begin{array}{l}\text { Placebo, then } \\
\text { octreotide } \\
\text { (Group 1) } \\
\text { vs octreotide, then } \\
\text { placebo (Group 2) } \\
\text { (all patients } \\
\text { received albumin) }\end{array}$ & $\begin{array}{l}20 \% \text { decrease } \\
\text { in SCr } \\
\text { after } 4 \mathrm{~d}\end{array}$ & $\begin{array}{l}\text { Group 1: } \\
215 \pm 32 \\
\mu \mathrm{mol} / \\
\text { Group 2: } 208 \\
\pm 16 \mu \mathrm{mol} / \mathrm{L}\end{array}$ & $\begin{array}{l}\text { Group } 1 \text { : } \\
222 \pm 41 \\
\mu \mathrm{mol} / \mathrm{L} \\
\text { after placebo; } \\
270 \pm 54 \\
\mu \mathrm{mol} / \mathrm{L} \\
\text { after octreotide } \\
\text { Group 2: } \\
194 \pm 34 \\
\mu \mathrm{mol} / \mathrm{L} \\
\text { after octreotide; } \\
204 \pm 47 \\
\mu \mathrm{mol} / \mathrm{L} \\
\text { after placebo }\end{array}$ & $\begin{array}{l}\text { Treatment with } \\
\text { octreotide was } \\
\text { not effective. }\end{array}$ & $\begin{array}{l}\text { The study } \\
\text { included types } 1 \\
\text { and } 2 \text { HRS patients } \\
\text { (numbers in each } \\
\text { group not } \\
\text { specified). } \\
\text { No side effects } \\
\text { reported. }\end{array}$ \\
\hline $\begin{array}{l}\text { Colle et al } \\
2002^{[42]}\end{array}$ & $18(18 / 0)$ & $\begin{array}{l}\text { Chart review } \\
\text { (retrospective } \\
\text { analysis) }\end{array}$ & $\begin{array}{l}\text { Terlipressin } \\
\text { (some patients } \\
\text { received albumin) }\end{array}$ & $\begin{array}{l}\text { Decrease in SCr } \\
\text { to }<130 \mu \mathrm{mol} / \mathrm{L} \\
\text { or decrease of at } \\
\text { least } 20 \% \text { leading } \\
\text { to a stable value; } \\
\text { evaluation of } \\
\text { predictive factors }\end{array}$ & $\begin{array}{l}\text { Patients with } \\
\text { improved } \\
\text { SCr: } \\
276 \pm 47 \\
\mu \mathrm{mol} / \mathrm{L}^{1} \\
\text { Patients } \\
\text { without } \\
\text { improved } \\
\text { SCr: } 295 \pm 89^{1} \\
\mu \mathrm{mol} / \mathrm{L}\end{array}$ & $\begin{array}{l}\text { Patients with } \\
\text { improved SCr: } \\
130 \pm 13 \\
\mu \mathrm{mol} / \mathrm{L} \\
\text { Patients with } \\
\text { improved SCr: } \\
411 \pm 89 \\
\mu \mathrm{mol} / \mathrm{L}\end{array}$ & $\begin{array}{l}11 \text { patients } \\
\text { had improved } \\
\text { renal function }\end{array}$ & $\begin{array}{l}\text { Some of these } \\
\text { patients were } \\
\text { included in the } \\
\text { Moreau study. } \\
\text { Patients with } \\
\text { improved renal } \\
\text { function had less } \\
\text { severe cirrhosis } \\
\text { than patients } \\
\text { without. Patients } \\
\text { without a } \\
\text { precipitating } \\
\text { factor for HRS } \\
\text { or who responded } \\
\text { to terlipressin } \\
\text { were more likely } \\
\text { to survive. }\end{array}$ \\
\hline $\begin{array}{l}\text { Halimi et al } \\
2002^{[41]}\end{array}$ & $18(16 / 2)$ & $\begin{array}{l}\text { Multicenter } \\
\text { pilot } \\
\text { (retrospective) }\end{array}$ & $\begin{array}{l}\text { Terlipressin } \\
\text { for } 2-16 \mathrm{~d}\end{array}$ & $\begin{array}{l}>30 \% \text { decrease } \\
\text { in baseline SCr }\end{array}$ & $\begin{array}{l}298 \pm 124 \\
\mu \mathrm{mol} / \mathrm{L}\end{array}$ & $\begin{array}{l}145 \pm 85 \\
\mu \mathrm{mol} / \mathrm{L}\end{array}$ & $\begin{array}{l}13 \text { of } 18 \text { had } \\
\text { improved renal } \\
\text { function; } 8 \text { had } \\
\text { a normal } \\
\text { SCr at d } 5\end{array}$ & $\begin{array}{l}\text { Three patients } \\
\text { had ischemic } \\
\text { side effects. } \\
\text { One had severe } \\
\text { bronchospasms } \\
\text { after terlipressin } \\
\text { administration, and } \\
\text { subsequently died. }\end{array}$ \\
\hline
\end{tabular}




\begin{tabular}{|c|c|c|c|c|c|c|c|c|}
\hline $\begin{array}{l}\text { Guevara et al } \\
1998^{[49]}\end{array}$ & 16 (Type NS) & $\begin{array}{l}\text { Open pilot } \\
\text { study }\end{array}$ & $\begin{array}{l}\text { Ornipressin } \\
\text { and albumin } \\
\text { for } 3 \text { vs } 15 \mathrm{~d}\end{array}$ & Efficacy & $\begin{array}{l}\text { 3-d arm: } \\
2.9 \pm 0.5 \\
\mathrm{mg} / \mathrm{dL} \\
15-\mathrm{d} \text { arm: } \\
3.0 \pm 0.5 \\
\mathrm{mg} / \mathrm{dL}\end{array}$ & $\begin{array}{l}3-\mathrm{d} \text { arm: } \\
2.2 \pm 0.4 \\
\mathrm{mg} / \mathrm{dL} \\
15-\mathrm{d} \text { arm: } \\
0.7 \pm 0.1 \\
\mathrm{mg} / \mathrm{dL}\end{array}$ & $\begin{array}{l}75 \% \text { of patients } \\
\text { had improved } \\
\text { renal function. }\end{array}$ & $\begin{array}{l}\text { Treatment was } \\
\text { stopped in } 4 \text { patients } \\
\text { on the } 15-\mathrm{d} \\
\text { protocol because } \\
\text { of ischemic } \\
\text { complications. }\end{array}$ \\
\hline $\begin{array}{l}\text { Angeli et al } \\
1999^{[62]}\end{array}$ & $13(13 / 0)$ & Nonrandomized & $\begin{array}{l}\text { Dopamine and } \\
\text { albumin (Group } \\
\text { A) vs midodrine, } \\
\text { octreotride, and IV } \\
\text { albumin (Group B) }\end{array}$ & Efficacy & $\begin{array}{l}\text { Group A: } \\
3.6 \pm 0.6 \\
\mathrm{mg} / \mathrm{dL} \\
\text { Group B: } \\
5.0 \pm 0.9 \\
\mathrm{mg} / \mathrm{dL}\end{array}$ & $\begin{array}{l}\text { Group A: } 5.1 \pm 1.5 \\
\mathrm{mg} / \mathrm{dL} \text { at } 15 \mathrm{~d} \\
\text { (only } 1 \text { patient } \\
\text { survived to } \mathrm{d} 20 \text { ) } \\
\text { Group B: } 3.3 \pm 0.7 \\
\mathrm{mg} / \mathrm{dL} \text { at } 20 \mathrm{~d}\end{array}$ & $\begin{array}{l}\text { All Group B patients } \\
\text { had improved GFR. } \\
7 \text { of } 8 \text { patients } \\
\text { in Group A had } \\
\text { worsening renal } \\
\text { function and died. }\end{array}$ & $\begin{array}{l}\text { No significant } \\
\text { side effects. }\end{array}$ \\
\hline $\begin{array}{l}\text { Holt et al } \\
1999^{[33]}\end{array}$ & 12 (NS) & Open label & $\begin{array}{l}\mathrm{N} \text {-acetylcysteine } \\
\text { for } 5 \mathrm{~d}\end{array}$ & Efficacy & $\begin{array}{l}222 \pm 27 \\
\mu \mathrm{mol} / \mathrm{L}\end{array}$ & $\begin{array}{l}169 \pm 7 \\
\mu \mathrm{mol} / \mathrm{L}\end{array}$ & & $\begin{array}{l}67 \% \text { survival at } \\
1 \text { mo, and } 58 \% \\
\text { at } 3 \text { mo ( } 2 \text { patients } \\
\text { received liver } \\
\text { transplants). }\end{array}$ \\
\hline $\begin{array}{l}\text { Mulkay et al } \\
2001^{[40]}\end{array}$ & $12(12 / 0)$ & Pilot & $\begin{array}{l}\text { Terlipressin for } \\
1 \mathrm{wk} \text { to } 2 \mathrm{mo}\end{array}$ & $\begin{array}{l}\text { Safety and } \\
\text { efficacy }\end{array}$ & $3.4 \mathrm{mg} / \mathrm{dL}$ & $1.8 \mathrm{mg} / \mathrm{dL}$ & & $\begin{array}{l}\text { Three patients } \\
\text { received liver } \\
\text { transplants, and } \\
\text { had near-normal } \\
\text { renal function. } \\
\text { The other } 9 \text { died } \\
\text { during follow-up. } \\
\text { No ischemic } \\
\text { complications. }\end{array}$ \\
\hline $\begin{array}{l}\text { Duvoux et al } \\
2002^{[48]}\end{array}$ & $12(12 / 0)$ & Pilot & $\begin{array}{l}\text { Noradrenalin } \\
\text { (NA), albumin, } \\
\text { and furosemide } \\
\text { for at least } 5 \mathrm{~d}\end{array}$ & $\begin{array}{l}\text { Safety and } \\
\text { efficacy }\end{array}$ & $\begin{array}{l}2.6 \pm 1.1 \\
\mathrm{mg} / \mathrm{dL} \text { pre- } \\
\text { furosemide/ } \\
\text { albumin; } \\
3.9 \pm 1.8 \\
\mathrm{mg} / \mathrm{dL} \\
\text { after infusion } \\
\text { (pre-NA) }\end{array}$ & $1.6 \pm 0.8 \mathrm{mg} / \mathrm{dL}$ & $\begin{array}{l}\text { Reversal of HRS } \\
\text { in } 10 \text { of } 12 \text { patients }\end{array}$ & $\begin{array}{l}\text { Two patients had } \\
\text { previously received } \\
\text { terlipressin } \\
\text { (underwent 48-h } \\
\text { washout before } \\
\text { starting NA). } \\
\text { Transient } \\
\text { myocardial } \\
\text { ischemia was } \\
\text { observed } \\
\text { in } 1 \text { patient. }\end{array}$ \\
\hline $\begin{array}{l}\text { Hadengue } \\
\text { et al } 1998^{[63]}\end{array}$ & $9(9 / 0)$ & $\begin{array}{l}\text { Double-blind, } \\
\text { short-term, } \\
\text { controlled } \\
\text { crossover } \\
\text { study }\end{array}$ & $\begin{array}{l}\text { Terlipressin and } \\
\text { placebo for } 2 \mathrm{~d} \text { in } \\
\text { randomized order }\end{array}$ & Efficacy & $\begin{array}{l}\text { Baseline } \mathrm{CrCl} \text { : } \\
15 \pm 2 \\
\mathrm{~mL} / \mathrm{min}\end{array}$ & $\begin{array}{l}\mathrm{CrCl} \text { after } \\
\text { terlipressin } \\
\text { (includes both } \\
\text { groups): } 27 \pm 4 \\
\mathrm{~mL} / \mathrm{min} \\
\mathrm{CrCl} \text { after placebo } \\
\text { (includes both } \\
\text { groups): } \\
16 \pm 3 \mathrm{~mL} / \mathrm{min}\end{array}$ & & $\begin{array}{l}\text { No side effects } \\
\text { reported. }\end{array}$ \\
\hline $\begin{array}{l}\text { Uriz et al } \\
2000^{[37]}\end{array}$ & $9(6 / 3)$ & Pilot & $\begin{array}{l}\text { Terlipressin with } \\
\text { albumin for } 5-15 \mathrm{~d}\end{array}$ & $\begin{array}{l}\text { Reduction } \\
\text { of serum } \\
\text { creatinine } \\
\text { to }<1.5 \\
\mathrm{mg} / \mathrm{dL}\end{array}$ & $\begin{array}{l}3.9 \pm 0.7 \\
\mathrm{mg} / \mathrm{dL}\end{array}$ & $1.5 \pm 0.2 \mathrm{mg} / \mathrm{dL}$ & $\begin{array}{l}\text { Reversal of HRS } \\
\text { in } 7 \text { of } 9 \text { patients. }\end{array}$ & $\begin{array}{l}\text { One patient did } \\
\text { not complete } \\
\text { the study due } \\
\text { to pancreatitis. } \\
\text { No ischemic } \\
\text { complications. }\end{array}$ \\
\hline $\begin{array}{l}\text { Angeli et al } \\
1998^{[45]}\end{array}$ & $\begin{array}{l}8(0 / 8)+ \\
17 \text { cirrhotic } \\
\text { patients } \\
\text { without HRS }\end{array}$ & Open label & $\begin{array}{l}\text { Midodrine } \\
\text { (one dose) }\end{array}$ & $\begin{array}{l}\text { Renal } \\
\text { function and } \\
\text { renal hemo- } \\
\text { dynamics } \\
\text { (acute effects) }\end{array}$ & $\begin{array}{l}\text { GFR: } \\
39.0 \pm 6.4 \\
\mathrm{~mL} / \mathrm{min}\end{array}$ & $\begin{array}{l}\text { GFR: } \\
45.1 \pm 7.6 \\
\mathrm{~mL} / \mathrm{min}\end{array}$ & $\begin{array}{l}\text { No significant acute } \\
\text { effect on renal } \\
\text { hemodynamics or } \\
\text { renal function. }\end{array}$ & $\begin{array}{l}\text { This study looked } \\
\text { at the acute effect } \\
\text { of one dose of } \\
\text { midodrine. } \\
\text { The results include } \\
\text { cirrhotic patients } \\
\text { without HRS. }\end{array}$ \\
\hline $\begin{array}{l}\text { Gulberg et al } \\
1999^{[64]}\end{array}$ & $7(7 / 0)$ & Nonrandomized & $\begin{array}{l}\text { Orinpressin, } \\
\text { dopamine, and } \\
\text { albumin for } 5-27 \mathrm{~d}\end{array}$ & $\begin{array}{l}2 \times \text { increase } \\
\text { in Crcl } \\
(\text { to }>40 \\
\mathrm{mL} / \mathrm{min})\end{array}$ & $\begin{array}{l}\text { Treatment } \\
\text { success } \\
\text { group: } \\
4.6 \pm 0.9 \\
\mathrm{mg} / \mathrm{dL} \text {, and } \\
\text { improved to } \\
1.3 \pm 0.2 \\
\mathrm{mg} / \mathrm{dL}\end{array}$ & $\begin{array}{l}\text { Treatment } \\
\text { success group: } \\
1.3 \pm 0.2 \\
\mathrm{mg} / \mathrm{dL}\end{array}$ & $\begin{array}{l}\text { HRS was reversed } \\
\text { in } 4 \text { of } 7 \text { patients }\end{array}$ & $\begin{array}{l}\text { Two responders } \\
\text { had a relapse. } \\
\text { One of them } \\
\text { responded to } \\
\text { retreatment, but } \\
\text { treatment was } \\
\text { stopped in the other } \\
\text { because of a } \\
\text { ventricular } \\
\text { tachyarrhythmia. } \\
\text { Treatment was } \\
\text { stopped in another } \\
\text { patient because of } \\
\text { intestinal ischemia. }\end{array}$ \\
\hline
\end{tabular}




\begin{tabular}{|c|c|c|c|c|c|c|c|}
\hline Kaffy et al & 5 (NS) & Pilot & Octreotide & Efficacy & $194 \mu \mathrm{mol} / \mathrm{L}$ & 96 ㅆmol/L & Improvement of but 4 of 5 patients eventually died. \\
\hline $1999^{[65]}$ & & & for $5 \mathrm{~d}$ & & in 4 patients & in 4 patients & $\begin{array}{ll}\text { SCr in } 4 \text { of } 5 & \text { HRS rapidly recurred when octreotide } \\
\text { patients, } & \text { was stopped, and did not respond to } \\
& \text { further octreotide infusion. }\end{array}$ \\
\hline
\end{tabular}

SCr: Serum Creatinine; RRT: Renal replacement therapy; NS: Not Specified; CrCl: Creatinine Clearance. Cr conversion from $\mu$ mol/L to mg/dL: divide by 88.4.

Table 4 Invasive therapies

\begin{tabular}{|c|c|c|c|c|c|c|c|c|}
\hline $\begin{array}{l}\text { Author and } \\
\text { Year }\end{array}$ & $\begin{array}{l}\text { N (\# Type } 1 \text {, } \\
\text { \# Type 2) }\end{array}$ & Study design & Intervention & $\begin{array}{l}\text { Outcome } \\
\text { measures }\end{array}$ & $\begin{array}{l}\text { Mean } \\
\text { baseline SCr }\end{array}$ & $\begin{array}{l}\text { Mean } \\
\text { follow-up SCr }\end{array}$ & Other results & Comments \\
\hline $\begin{array}{l}\text { Brensing et al } \\
2000^{[50]}\end{array}$ & $\begin{array}{l}31(14 / 17) ; \text { an } \\
\text { additional } 10 \\
\text { were too sick } \\
\text { to receive TIPS }\end{array}$ & Phase II & TIPS & $\begin{array}{l}\text { Safety and } \\
\text { survival }\end{array}$ & $\begin{array}{l}\text { (Of the } 31 \\
\text { patients who } \\
\text { received TIPS) } \\
2.3 \pm 1.7 \\
\mathrm{mg} / \mathrm{dL}\end{array}$ & $\begin{array}{l}\text { Wk 4: } \\
1.5 \pm 1.2 \\
\mathrm{mg} / \mathrm{dL}\end{array}$ & $\begin{array}{l}\text { Renal function } \\
\text { improved within } \\
2 \text { wk after } \\
\text { TIPS and } \\
\text { subsequently } \\
\text { stabilized. }\end{array}$ & $\begin{array}{l}\text { Three-month } \\
\text { survival rate was } \\
81 \% \text { ( } 10 \% \text { of non- } \\
\text { shunted patients } \\
\text { survived, but } \\
\text { they were felt } \\
\text { to be too sick to } \\
\text { receive TIPS). } \\
\text { There was } 1 \\
\text { TIPS-related death. }\end{array}$ \\
\hline $\begin{array}{l}\text { Wong et al } \\
2004^{[46]}\end{array}$ & $14(14 / 0)$ & Prospective & $\begin{array}{l}\text { Midodrine, } \\
\text { octreotide, } \\
\text { albumin, } \\
\text { and TIPS }\end{array}$ & $\begin{array}{l}\text { Efficacy (serum } \\
\text { creatinine }<135 \\
\mu \mathrm{mol} / \mathrm{L} \text { for } \\
\text { at least } 3 \mathrm{~d} \text { ) }\end{array}$ & $\begin{array}{l}\text { Responders: } \\
233 \pm 29 \\
\mu \mathrm{mol} / \mathrm{L} \\
\text { Nonresponders: } \\
345 \pm 83 \\
\mu \mathrm{mol} / \mathrm{L}\end{array}$ & $\begin{array}{l}\text { Responders: } \\
112 \pm 8 \\
\mu \text { mol/L after } \\
\text { medical therapy } \\
\text { Nonresponders: } \\
476 \pm 139 \\
\mu \text { mol/L after } \\
\text { medical therapy. }\end{array}$ & $\begin{array}{l}\text { Renal function } \\
\text { improved in } 10 \\
\text { of } 14 \text { patients } \\
\text { ( } 71 \% \text { ) with } \\
\text { medical therapy. } \\
\text { Five responders } \\
\text { received } \\
\text { TIPS; their } \\
\text { renal function } \\
\text { continued to } \\
\text { improve. Mean } \\
\text { GFR was } 96 \pm 20 \\
\text { mL/min by } 12 \\
\text { mo post-TIPS. }\end{array}$ & $\begin{array}{l}\text { TIPS was } \\
\text { performed in } \\
\text { responders who } \\
\text { were stable. } \\
\text { Two of the five } \\
\text { responders } \\
\text { who did not } \\
\text { receive TIPS } \\
\text { underwent liver } \\
\text { transplantation, } \\
\text { and their SCr } \\
\text { remained } \\
\text { normal at the } \\
\text { time of liver } \\
\text { transplantation. }\end{array}$ \\
\hline $\begin{array}{l}\text { Alessandria } \\
\text { et al } 2002^{[36]}\end{array}$ & $\begin{array}{l}16(0 / 11 \text {, and } \\
\text { an additional } 5 \\
\text { with "organic } \\
\text { renal disease") }\end{array}$ & $\begin{array}{l}\text { Prospective, } \\
\text { nonrandomized }\end{array}$ & $\begin{array}{l}\text { Terlipressin } \\
\text { for } 7 \mathrm{~d} \text { (and } \\
\text { TIPS in stable } \\
\text { patients) }\end{array}$ & Efficacy & $\begin{array}{l}2.4 \pm 0.9 \\
\mathrm{mg} / \mathrm{dL}\end{array}$ & $\begin{array}{l}\text { After terlipressin } \\
\text { therapy: } 1.8 \pm 0.8 \\
\text { mg/dL } \\
\text { After TIPS: } \\
1.4 \pm 0.3 \\
\mathrm{mg} / \mathrm{dL}\end{array}$ & $\begin{array}{l}\text { Terlipressin: } 8 \text { of } \\
11 \text { HRS patients } \\
\text { had improved } \\
\text { renal function } \\
\text { (and } 7 \text { of the } 8 \\
\text { responders had } \\
\text { reversal of HRS } \\
\text { (SCr }<1.5 \mathrm{mg} / \mathrm{dL} \text { ) } \\
\text { Subsequent TIPS: } \\
8 \text { of } 9 \text { patients } \\
\text { ( } 89 \% \text { ) who } \\
\text { underwent TIPS } \\
\text { had improved } \\
\text { renal function by } \\
1 \text { mo. }\end{array}$ & $\begin{array}{l}\text { Renal function } \\
\text { improved } \\
\text { significantly } \\
\text { after TIPS in all } \\
\text { patients who } \\
\text { responded to } \\
\text { terlipressin. } \\
\text { One HRS patient } \\
\text { who did not } \\
\text { respond to } \\
\text { terlipressin } \\
\text { underwent TIPS } \\
\text { and responded. } \\
\text { In the non-HRS } \\
\text { group (with } \\
\text { "organic renal } \\
\text { disease", only } \\
\text { one patient had } \\
\text { an improved } \\
\text { SCr (from } 3.7 \\
\text { to } 1.8 \text { mg/dL) } \\
\text { with terlipressin } \\
\text { treatment. }\end{array}$ \\
\hline $\begin{array}{l}\text { Guevara et al } \\
1998^{[49]}\end{array}$ & $7(7 / 0)$ & Prospective & TIPS & Efficacy & $\begin{array}{l}4.9 \pm 0.8 \\
\mathrm{mg} / \mathrm{dL}\end{array}$ & $\begin{array}{l}1 \mathrm{wk} \text { after TIPS: } \\
3.7 \pm 1.0 \mathrm{mg} / \mathrm{dL} \\
1 \mathrm{mo} \text { after TIPS: } \\
1.8 \pm 0.4 \mathrm{mg} / \mathrm{dL}\end{array}$ & $\begin{array}{l}\text { Renal function } \\
\text { improved in } 6 \text { of } \\
7 \text { patients. }\end{array}$ & $\begin{array}{l}\text { Mean survival } \\
\text { was } 4.7 \pm 2 \text { mo. }\end{array}$ \\
\hline $\begin{array}{l}\text { Witzke et al } \\
2004^{[53]}\end{array}$ & 30 (NS) & Prospective & $\begin{array}{l}\text { CVVHD (if } \\
\text { mechanically } \\
\text { ventilated) vs } \\
\text { intermittent } \\
\text { HD if not } \\
\text { ventilated }\end{array}$ & Survival & $\mathrm{N} / \mathrm{A}$ & $\mathrm{N} / \mathrm{A}$ & $\begin{array}{l}8 \text { of } 15 \text { patients } \\
\text { who received HD } \\
\text { survived. None } \\
\text { of the ventilated } \\
\text { patients (received } \\
\text { CVVHD) survived. }\end{array}$ & $\begin{array}{l}\text { Note that the } \\
\text { sickest patients } \\
\text { (on a ventilator) } \\
\text { all received } \\
\text { CVVHD. }\end{array}$ \\
\hline
\end{tabular}




\begin{tabular}{|c|c|c|c|c|c|c|c|c|}
\hline $\begin{array}{l}\text { Keller et al } \\
1995^{[54]}\end{array}$ & $\begin{array}{l}26(\mathrm{NS}) ; \text { an } \\
\text { additional } 81 \\
\text { patients had } \\
\text { liver disease } \\
\text { and renal } \\
\text { failure, } \\
\text { but were } \\
\text { not diagnosed } \\
\text { with HRS }\end{array}$ & Retrospective & HD & $\begin{array}{l}\text { Risk factor } \\
\text { evaluation } \\
\text { and outcomes }\end{array}$ & $\mathrm{N} / \mathrm{A}$ & $\mathrm{N} / \mathrm{A}$ & $\begin{array}{l}7 \text { of } 16 \text { patients } \\
\text { with HRS who } \\
\text { received HD } \\
\text { survived, while } \\
\text { only } 1 \text { out of } 16 \\
\text { patients with } \\
\text { HRS who did } \\
\text { not receive HD } \\
\text { survived. }\end{array}$ & \\
\hline $\begin{array}{l}\text { Mitzner et al } \\
2000^{[55]}\end{array}$ & 13 (Type 1) & $\begin{array}{l}\text { Prospective, } \\
\text { randomized, } \\
\text { controlled }\end{array}$ & $\begin{array}{l}\text { MARS and } \\
\text { HDF and } \\
\text { standard medical } \\
\text { therapy } v \text { HDF } \\
\text { and medical } \\
\text { therapy }\end{array}$ & Survival & $\begin{array}{l}\text { MARS + HDF: } \\
3.8 \pm 1.5 \\
\text { mg/dL } \\
\text { HDF alone: } \\
4.4 \pm 1.3 \\
\mathrm{mg} / \mathrm{dL}\end{array}$ & $\begin{array}{l}\text { MARS + HDF: } \\
2.3 \pm 1.5 \mathrm{mg} / \mathrm{dL} \\
\text { HDF alone: } \\
3.8 \pm 0.5 \mathrm{mg} / \mathrm{dL}\end{array}$ & $\begin{array}{l}\text { At one week: } \\
62.5 \% \text { mortality } \\
\text { in the treatment } \\
\text { group, and } 100 \% \\
\text { mortality in } \\
\text { the group who } \\
\text { did not receive } \\
\text { MARS. }\end{array}$ & $\begin{array}{l}\text { None of these } \\
\text { patients } \\
\text { underwent liver } \\
\text { transplantation } \\
\text { or received } \\
\text { TIPS or } \\
\text { vasopressin } \\
\text { analogues during } \\
\text { the observation } \\
\text { period. }\end{array}$ \\
\hline $\begin{array}{l}\text { Jalan et al } \\
2003^{[66]}\end{array}$ & $\begin{array}{l}8(5 / 2 \text {, and } \\
\text { one patient } \\
\text { without } \\
\text { HRS) }\end{array}$ & $\begin{array}{l}\text { Prospective, } \\
\text { nonrandomized }\end{array}$ & MARS & $\begin{array}{l}\text { Safety and } \\
\text { efficacy }\end{array}$ & $\begin{array}{l}162(51-312) \\
\mu \mathrm{mol} / \mathrm{L}\end{array}$ & $\begin{array}{l}108 \text { (34-231) } \\
\mu \mathrm{mol} / \mathrm{L}\end{array}$ & $\begin{array}{l}50 \% \text { survival at } \\
3 \text { mo follow-up }\end{array}$ & $\begin{array}{l}\text { All of the patients } \\
\text { had alcoholic } \\
\text { hepatitis and were } \\
\text { encephalopathic. } \\
\text { Renal function } \\
\text { improved in all } \\
\text { patients. Of the } \\
5 \text { patients with } \\
\text { type } 1 \text { HRS, } 3 \\
\text { remained anuric, } \\
\text { but there was } \\
\text { normalization of } \\
\text { SCr in the other } \\
2 \text { patients. SCr } \\
\text { was normalized } \\
\text { in both patients } \\
\text { with type } 2 \text { HRS } \\
\text { by the end of } \\
\text { treatment. }\end{array}$ \\
\hline $\begin{array}{l}\text { Mitzner et al } \\
2001^{[55]}\end{array}$ & 8 (NS) & Uncontrolled & MARS & $\begin{array}{l}\text { Multiple } \\
\text { organ } \\
\text { function } \\
\text { changes }\end{array}$ & $\begin{array}{l}380 \pm 182 \\
\mu \mathrm{mol} / \mathrm{L}\end{array}$ & $\begin{array}{l}163 \pm 119 \\
\mu \mathrm{mol} / \mathrm{L}\end{array}$ & $\begin{array}{l}\text { Improvement in } \\
\text { multiple organ } \\
\text { functions }\end{array}$ & \\
\hline
\end{tabular}

SCr: Serum creatinine; $\mathrm{CrCl}$ : Creatinine clearance; TIPS: Transjugular intrahepatic portosystemic shunt; NS: Not Specified; CVVHD: Continuous veno-venous hemodialysis; HD: Hemodialysis; MARS: Molecular adsorbents recirculating system; HDF: Hemodiafiltration. Cr conversion from $\mu$ mol/L to mg/dL: divide by 88.4 .

for use in North America, but a double-blind, randomized, placebo-controlled trial is now being conducted in the USA and Germany in patients with type 1 HRS. Alpha-1 adrenoreceptor agonists and a somatostatin analogue are readily available in North America and have been studied in type $1 \mathrm{HRS}$. An observational study compared vasopressin infusion with octeotride infusion in patients with HRS, and found a complete response rate of $41 \%$ in the patients treated with vasopressin compared with $0 \%$ in the patients treated with octreotide ${ }^{[44]}$. In type $1 \mathrm{HRS}$, alpha-1 agonists have only been used in combination with other agents. Few nonrandomized, prospective studies have evaluated treatment with both midodrine and octreotide ${ }^{[45-47]}$. The study by Angeli ${ }^{[45]}$ included only five patients and showed that after $20 \mathrm{~d}$ of treatment, all patients had serum creatinine levels below $2 \mathrm{mg} / \mathrm{dL}$. In the study by Wong et al ${ }^{[4]}$, 10 of 14 patients with HRS treated with midodrine, octreotide, and albumin had their serum creatinine stable at less than $1.5 \mathrm{mg} / \mathrm{dL}$ for three days. The use of noradrenalin in combination with intravenous albumin and furosemide was studied in 12 patients $^{[48]}$. HRS was reversed in $83 \%$ of patients, with an adverse event rate of $17 \%$. These small studies suggest a short-term benefit in improving renal function in HRS patients, although larger, randomized studies are required before recommending the routine use of these agents in clinical practice. Other drugs, such as $\mathrm{N}$-acetylcysteine and misoprostol, have been proposed as therapy for HRS, but have not been well-studied.

\section{Non-pharmacologic therapy}

Transjugular intrahepatic portosystemic shunts (TIPS), by reducing portal hypertension, may be useful in treating HRS (Table 4), although no trials have shown a survival advantage ${ }^{[49-51]}$.

\section{Renal replacement therapy}

Patients with HRS who progress to severe renal failure can be initiated on renal replacement therapy (RRT), generally given as continuous hemofiltration. Dialysis 
is usually used as a bridge in patients who are awaiting liver transplantation, and is not recommended for patients who are unlikely to recover from liver failure or are unlikely to receive liver transplantation because of other contraindications. Survival on dialysis is generally dependent on the severity of liver disease ${ }^{[52]}$. There are only a few small studies evaluating the effects of dialysis in HRS $^{[53,54]}$. Keller et a ${ }^{54]}$, in a retrospective study, found that 7 of 16 patients with HRS who received RRT survived, while only 1 out of 16 patients with HRS who did not receive RRT survived. In the prospective study by Witzke et at ${ }^{[53]}, 30$ patients with Child-Pugh C cirrhosis and HRS were treated with continuous veno-venous hemodialysis (CVVHD) if they were on mechanical ventilation, or with intermittent hemodialysis if they were not on mechanical ventilation. No patients on mechanical ventilation survived for more than $30 \mathrm{~d}$, but 8 of 15 patients who were not on mechanical ventilation survived for more than $30 \mathrm{~d}$. The absence of a control group and lack of randomization make it difficult to draw any firm conclusions from this study.

Molecular absorbent recirculating system (MARS) is a form of albumin dialysis, which removes "toxins" such as tumor necrosis factor-alpha, interleukin-6, and nitric oxide via binding to dialysate albumin. A small, randomized trial showed a survival advantage of MARS when compared to standard therapy in HRS patients ${ }^{[5]}$. To date, there have been no other published trials comparing MARS to standard RRT.

\section{Transplantation}

Liver transplantation (LT) is the only effective and permanent treatment for $\operatorname{HRS}^{[10,14,56,57]}$ that cures both the liver and renal failure. However, the 5-year survival rate in LT recipients with HRS is significantly less than in LT patients without HRS $^{[56]}$. Patients who undergo LT may sometimes require postoperative hemodialysis. It is preferable to delay administration of cyclosporine or tacrolimus until renal impairment improves in these patients, as these drugs may further worsen renal function. The issue of whether to transplant a kidney in addition to a liver (LKT, combined liver kidney transplantation) is an important one as well. HRS alone is not considered an indication for a $\mathrm{LKT}^{[58]}$. A renal biopsy may be helpful in some patients to identify the etiology of the renal failure and to determine the presence and extent of glomerular scarring ${ }^{[59]}$. LKT should be reserved for patients with irreversible renal failure, including HRS patients who are on dialysis for more than 8 wk or patients with progressive primary renal disease ${ }^{[59]}$. United Network of Organ Sharing (UNOS) data indicate a 5 -year survival of LKT recipients of $62 \%$ compared with $50 \%$ for patients with a serum creatinine $>2.0 \mathrm{mg} / \mathrm{dL}$ receiving isolated LT $(P=0.0001)$. One center's results demonstrated a 5 -year patient survival of $48 \%$ for LKT patients, $67 \%$ for HRS patients receiving isolated LT, and $70 \%$ for patients with a serum creatinine $>2.0 \mathrm{mg} / \mathrm{dL}$ receiving isolated $\mathrm{LT}$ ( $P$ not significant comparing all groups $)^{[58]}$. It is not clear if the advances in management of HRS in recent years have had an impact on post-transplant outcomes. In a case-control study by Restuccia et al, patients with HRS treated with vasopressors and albumin prior to LT had similar survival outcomes compared to those patients who underwent OLT without HRS $^{[60]}$. However, the study had only 9 patients with HRS and as correctly stated by the authors, further confirmation in a larger series of patients is required. Clearly, further prospective studies are needed to guide transplant physicians to determine whether they should transplant the liver and the kidney or the liver alone in patients with liver failure and kidney failure.

\section{CONCLUSION}

Renal failure occurs commonly in patients with severe liver disease and its causes are multifactorial. Patients with type 1 HRS generally have a fatal outcome without expedited liver transplantation. Therapy with terlipressin and albumin looks promising, but there is a paucity of data to make firm conclusions. Use of other vasoconstrictors or TIPS remains experimental. The only proven treatment option is expedited liver transplantation. Dialysis is often used as a bridge to liver transplantation, but there are no controlled studies to support renal replacement therapy in type 1 HRS. Further research is necessary to better elucidate the mechanisms of HRS and to identify treatment strategies to reduce morbidity and mortality in patients with liver disease.

\section{REFERENCES}

1 Hecker R, Sherlock S. Electrolyte and circulatory changes in terminal liver failure. Lancet 1956; 271: 1121-1125

2 Arroyo V, Gines P, Gerbes AL, Dudley FJ, Gentilini P, Laffi G, Reynolds TB, Ring-Larsen H, Scholmerich J. Definition and diagnostic criteria of refractory ascites and hepatorenal syndrome in cirrhosis. International Ascites Club. Hepatology 1996; 23: 164-176

3 Baldus WP, Feichter RN, Summerskill WH. The Kidney in Cirrhosis. I. Clinical and Biochemical Features of Azotemia in Hepatic Failure. Ann Intern Med 1964; 60: 353-365

4 Baldus WP, Feichter RN, Summerskill WH, Hunt JC, Wakim KG. The Kidney in Cirrhosis. II. Disorders of Renal Function. Ann Intern Med 1964; 60: 366-377

5 Shear L, Kleinerman J, Gabuzda GJ. Renal Failure in Patients with Cirrhosis of the Liver. I. Clinical and Pathologic Characteristics. Am J Med 1965; 39: 184-198

6 Iwatsuki S, Popovtzer MM, Corman JL, Ishikawa M, Putnam CW, Katz FH, Starzl TE. Recovery from "hepatorenal syndrome" after orthotopic liver transplantation. N Engl J Med 1973; 289: 1155-1159

7 Gines A, Escorsell A, Gines P, Salo J, Jimenez W, Inglada L, Navasa M, Claria J, Rimola A, Arroyo V. Incidence, predictive factors, and prognosis of the hepatorenal syndrome in cirrhosis with ascites. Gastroenterology 1993; 105: 229-236

8 Wilkinson SP, Blendis LM, Williams R. Frequency and type of renal and electrolyte disorders in fulminant hepatic failure. $\mathrm{Br}$ Med J 1974; 1: 186-189

9 Platt JF, Ellis JH, Rubin JM, Merion RM, Lucey MR. Renal duplex Doppler ultrasonography: a noninvasive predictor of kidney dysfunction and hepatorenal failure in liver disease. Hepatology 1994; 20: 362-369

10 Dagher L, Moore K. The hepatorenal syndrome. Gut 2001; 49: 729-737

11 Epstein M, Berk DP, Hollenberg NK, Adams DF, Chalmers TC, Abrams HL, Merrill JP. Renal failure in the patient with cirrhosis. The role of active vasoconstriction. Am J Med 1970; 49: 175-185

12 Schroeder ET, Shear L, Sancetta SM, Gabuzda GJ. Renal failure in patients with cirrhosis of the liver. 3. Evaluation of 
intrarenal blood flow by para-aminohippurate extraction and response to angiotensin. Am J Med 1967; 43: 887-896

13 Moore K. The hepatorenal syndrome. Clin Sci (Lond) 1997; 92: 433-443

14 Gines P, Guevara M, Arroyo V, Rodes J. Hepatorenal syndrome. Lancet 2003; 362: 1819-1827

15 Martin PY, Gines P, Schrier RW. Nitric oxide as a mediator of hemodynamic abnormalities and sodium and water retention in cirrhosis. N Engl J Med 1998; 339: 533-541

16 Arroyo V, Gines P, Rimola A, Gaya J. Renal function abnormalities, prostaglandins, and effects of nonsteroidal antiinflammatory drugs in cirrhosis with ascites. An overview with emphasis on pathogenesis. Am J Med 1986; 81: 104-122

17 Arroyo V, Planas R, Gaya J, Deulofeu R, Rimola A, PerezAyuso RM, Rivera F, Rodes J. Sympathetic nervous activity, renin-angiotensin system and renal excretion of prostaglandin E2 in cirrhosis. Relationship to functional renal failure and sodium and water excretion. Eur J Clin Invest 1983; 13: 271-278

18 Schrier RW, Arroyo V, Bernardi M, Epstein M, Henriksen JH, Rodes J. Peripheral arterial vasodilation hypothesis: a proposal for the initiation of renal sodium and water retention in cirrhosis. Hepatology 1988; 8: 1151-1157

19 Arroyo V, Claria J, Salo J, Jimenez W. Antidiuretic hormone and the pathogenesis of water retention in cirrhosis with ascites. Semin Liver Dis 1994; 14: 44-58

20 Davidson EW, Dunn MJ. Pathogenesis of the hepatorenal syndrome. Annu Rev Med 1987; 38: 361-372

21 Ruiz-del-Arbol L, Monescillo A, Arocena C, Valer P, Gines P, Moreira V, Milicua JM, Jimenez W, Arroyo V. Circulatory function and hepatorenal syndrome in cirrhosis. Hepatology 2005; 42: 439-447

22 Ruiz-del-Arbol L, Urman J, Fernandez J, Gonzalez M, Navasa M, Monescillo A, Albillos A, Jimenez W, Arroyo V. Systemic, renal, and hepatic hemodynamic derangement in cirrhotic patients with spontaneous bacterial peritonitis. Hepatology 2003; 38: 1210-1218

23 Cardenas A. Hepatorenal syndrome: a dreaded complication of end-stage liver disease. Am J Gastroenterol 2005; 100: 460-467

24 Arroyo V. Physiopathology of refractory ascites and the hepatorenal syndrome. Nefrologia 2002; 22 Suppl 5: 41-46

25 Follo A, Llovet JM, Navasa M, Planas R, Forns X, Francitorra A, Rimola A, Gassull MA, Arroyo V, Rodes J. Renal impairment after spontaneous bacterial peritonitis in cirrhosis: incidence, clinical course, predictive factors and prognosis. Hepatology 1994; 20: 1495-1501

26 Sort P, Navasa M, Arroyo V, Aldeguer X, Planas R, Ruiz-delArbol L, Castells L, Vargas V, Soriano G, Guevara M, Gines P, Rodes J. Effect of intravenous albumin on renal impairment and mortality in patients with cirrhosis and spontaneous bacterial peritonitis. $N$ Engl J Med 1999; 341: 403-409

27 Gines P, Tito L, Arroyo V, Planas R, Panes J, Viver J, Torres M, Humbert P, Rimola A, Llach J. Randomized comparative study of therapeutic paracentesis with and without intravenous albumin in cirrhosis. Gastroenterology 1988; 94: 1493-1502

28 Cardenas A, Gines P, Uriz J, Bessa X, Salmeron JM, Mas A, Ortega R, Calahorra B, De Las Heras D, Bosch J, Arroyo V, Rodes J. Renal failure after upper gastrointestinal bleeding in cirrhosis: incidence, clinical course, predictive factors, and short-term prognosis. Hepatology 2001; 34: 671-676

29 Caregaro L, Menon F, Angeli P, Amodio P, Merkel C, Bortoluzzi A, Alberino F, Gatta A. Limitations of serum creatinine level and creatinine clearance as filtration markers in cirrhosis. Arch Intern Med 1994; 154: 201-205

30 Maroto A, Gines A, Salo J, Claria J, Gines P, Anibarro L, Jimenez W, Arroyo V, Rodes J. Diagnosis of functional kidney failure of cirrhosis with Doppler sonography: prognostic value of resistive index. Hepatology 1994; 20: 839-844

31 Akriviadis E, Botla R, Briggs W, Han S, Reynolds T, Shakil O. Pentoxifylline improves short-term survival in severe acute alcoholic hepatitis: a double-blind, placebo-controlled trial. Gastroenterology 2000; 119: 1637-1648

32 Soper CP, Latif AB, Bending MR. Amelioration of hepatorenal syndrome with selective endothelin-A antagonist. Lancet 1996;

\section{7: 1842-1843}

33 Holt S, Goodier D, Marley R, Patch D, Burroughs A, Fernando B, Harry D, Moore K. Improvement in renal function in hepatorenal syndrome with N-acetylcysteine. Lancet 1999; 353 294-295

34 Moreau R, Lebrec D. The use of vasoconstrictors in patients with cirrhosis: type 1 HRS and beyond. Hepatology 2006; 43 385-394

35 Guevara M, Gines P, Fernandez-Esparrach G, Sort P, Salmeron JM, Jimenez W, Arroyo V, Rodes J. Reversibility of hepatorenal syndrome by prolonged administration of ornipressin and plasma volume expansion. Hepatology 1998; 27: 35-41

36 Alessandria C, Venon WD, Marzano A, Barletti C, Fadda M, Rizzetto M. Renal failure in cirrhotic patients: role of terlipressin in clinical approach to hepatorenal syndrome type 2. Eur J Gastroenterol Hepatol 2002; 14: 1363-1368

37 Uriz J, Gines P, Cardenas A, Sort P, Jimenez W, Salmeron JM, Bataller R, Mas A, Navasa M, Arroyo V, Rodes J. Terlipressin plus albumin infusion: an effective and safe therapy of hepatorenal syndrome. J Hepatol 2000; 33: 43-48

38 Moreau R, Durand F, Poynard T, Duhamel C, Cervoni JP, Ichai P, Abergel A, Halimi C, Pauwels M, Bronowicki JP, Giostra E, Fleurot C, Gurnot D, Nouel O, Renard P, Rivoal M, Blanc P, Coumaros D, Ducloux S, Levy S, Pariente A, Perarnau JM, Roche J, Scribe-Outtas M, Valla D, Bernard B, Samuel D, Butel J, Hadengue A, Platek A, Lebrec D, Cadranel JF. Terlipressin in patients with cirrhosis and type 1 hepatorenal syndrome: a retrospective multicenter study. Gastroenterology 2002; 122: 923-930

39 Ortega R, Gines P, Uriz J, Cardenas A, Calahorra B, De Las Heras D, Guevara M, Bataller R, Jimenez W, Arroyo V, Rodes J. Terlipressin therapy with and without albumin for patients with hepatorenal syndrome: results of a prospective, nonrandomized study. Hepatology 2002; 36: 941-948

40 Mulkay JP, Louis H, Donckier V, Bourgeois N, Adler M, Deviere J, Le Moine O. Long-term terlipressin administration improves renal function in cirrhotic patients with type 1 hepatorenal syndrome: a pilot study. Acta Gastroenterol Belg 2001; 64: 15-19

41 Halimi C, Bonnard P, Bernard B, Mathurin P, Mofredj A, di Martino V, Demontis R, Henry-Biabaud E, Fievet P, Opolon P, Poynard T, Cadranel JF. Effect of terlipressin (Glypressin) on hepatorenal syndrome in cirrhotic patients: results of a multicentre pilot study. Eur J Gastroenterol Hepatol 2002; 14: 153-158

42 Colle I, Durand F, Pessione F, Rassiat E, Bernuau J, Barriere E, Lebrec D, Valla DC, Moreau R. Clinical course, predictive factors and prognosis in patients with cirrhosis and type 1 hepatorenal syndrome treated with terlipressin: a retrospective analysis. J Gastroenterol Hepatol 2002; 17: 882-888

43 Solanki P, Chawla A, Garg R, Gupta R, Jain M, Sarin SK. Beneficial effects of terlipressin in hepatorenal syndrome: a prospective, randomized placebo-controlled clinical trial. J Gastroenterol Hepatol 2003; 18: 152-156

44 Kiser TH, Fish DN, Obritsch MD, Jung R, MacLaren R, Parikh CR. Vasopressin, not octreotide, may be beneficial in the treatment of hepatorenal syndrome: a retrospective study. Nephrol Dial Transplant 2005; 20: 1813-1820

45 Angeli P, Volpin R, Piovan D, Bortoluzzi A, Craighero R, Bottaro S, Finucci GF, Casiglia E, Sticca A, De Toni R, Pavan L, Gatta A. Acute effects of the oral administration of midodrine, an alpha-adrenergic agonist, on renal hemodynamics and renal function in cirrhotic patients with ascites. Hepatology 1998; 28: 937-943

46 Wong F, Pantea L, Sniderman K. Midodrine, octreotide, albumin, and TIPS in selected patients with cirrhosis and type 1 hepatorenal syndrome. Hepatology 2004; 40: 55-64

47 Kalambokis G, Economou M, Fotopoulos A, Al Bokharhii J, Pappas C, Katsaraki A, Tsianos EV. The effects of chronic treatment with octreotide versus octreotide plus midodrine on systemic hemodynamics and renal hemodynamics and function in nonazotemic cirrhotic patients with ascites. Am J Gastroenterol 2005; 100: 879-885 
48 Duvoux C, Zanditenas D, Hezode C, Chauvat A, Monin JL, Roudot-Thoraval F, Mallat A, Dhumeaux D. Effects of noradrenalin and albumin in patients with type I hepatorenal syndrome: a pilot study. Hepatology 2002; 36: 374-380

49 Guevara M, Gines P, Bandi JC, Gilabert R, Sort P, Jimenez W, Garcia-Pagan JC, Bosch J, Arroyo V, Rodes J. Transjugular intrahepatic portosystemic shunt in hepatorenal syndrome: effects on renal function and vasoactive systems. Hepatology 1998; 28: 416-422

50 Brensing KA, Textor J, Perz J, Schiedermaier P, Raab P, Strunk H, Klehr HU, Kramer HJ, Spengler U, Schild H, Sauerbruch T. Long term outcome after transjugular intrahepatic portosystemic stent-shunt in non-transplant cirrhotics with hepatorenal syndrome: a phase II study. Gut 2000; 47: 288-295

51 Ochs A. Transjugular intrahepatic portosystemic shunt. Dig Dis 2005; 23: 56-64

52 Wilkinson SP, Williams R. Renal failure in cirrhosis: current views and speculations. Adv Nephrol Necker Hosp 1977; 7: 15-32

53 Witzke O, Baumann M, Patschan D, Patschan S, Mitchell A, Treichel U, Gerken G, Philipp T, Kribben A. Which patients benefit from hemodialysis therapy in hepatorenal syndrome? J Gastroenterol Hepatol 2004; 19: 1369-1373

54 Keller F, Heinze H, Jochimsen F, Passfall J, Schuppan D, Buttner P. Risk factors and outcome of 107 patients with decompensated liver disease and acute renal failure (including 26 patients with hepatorenal syndrome): the role of hemodialysis. Ren Fail 1995; 17: 135-146

55 Mitzner SR, Stange J, Klammt S, Risler T, Erley CM, Bader BD, Berger ED, Lauchart W, Peszynski P, Freytag J, Hickstein H, Loock J, Lohr JM, Liebe S, Emmrich J, Korten G, Schmidt R. Improvement of hepatorenal syndrome with extracorporeal albumin dialysis MARS: results of a prospective, randomized, controlled clinical trial. Liver Transpl 2000; 6: 277-286

56 Gonwa TA, Morris CA, Goldstein RM, Husberg BS, Klintmalm GB. Long-term survival and renal function following liver transplantation in patients with and without hepatorenal syndrome--experience in 300 patients. Transplantation 1991; 51: $428-430$
57 Le Moine O. Hepatorenal syndrome--outcome after liver transplantation. Nephrol Dial Transplant 1998; 13: 20-22

58 Jeyarajah DR, Gonwa TA, McBride M, Testa G, Abbasoglu O, Husberg BS, Levy MF, Goldstein RM, Klintmalm GB. Hepatorenal syndrome: combined liver kidney transplants versus isolated liver transplant. Transplantation 1997; 64: 1760-1765

59 Davis CL. Impact of pretransplant renal failure: when is listing for kidney-liver indicated? Liver Transpl 2005; 11: S35-S44

60 Restuccia T, Ortega R, Guevara M, Gines P, Alessandria C, Ozdogan O, Navasa M, Rimola A, Garcia-Valdecasas JC, Arroyo V, Rodes J. Effects of treatment of hepatorenal syndrome before transplantation on posttransplantation outcome. A case-control study. J Hepatol 2004; 40: 140-146

61 Pomier-Layrargues G, Paquin SC, Hassoun Z, Lafortune M, Tran A. Octreotide in hepatorenal syndrome: a randomized, double-blind, placebo-controlled, crossover study. Hepatology 2003; 38: 238-243

62 Angeli P, Volpin R, Gerunda G, Craighero R, Roner P, Merenda R, Amodio P, Sticca A, Caregaro L, Maffei-Faccioli A, Gatta A. Reversal of type 1 hepatorenal syndrome with the administration of midodrine and octreotide. Hepatology 1999; 29: 1690-1697

63 Hadengue A, Gadano A, Moreau R, Giostra E, Durand F, Valla D, Erlinger S, Lebrec D. Beneficial effects of the 2-day administration of terlipressin in patients with cirrhosis and hepatorenal syndrome. J Hepatol 1998; 29: 565-570

64 Gulberg V, Bilzer M, Gerbes AL. Long-term therapy and retreatment of hepatorenal syndrome type 1 with ornipressin and dopamine. Hepatology 1999; 30: 870-875

65 Kaffy F, Borderie C, Chagneau C, Ripault MP, Larzilliere I, Silvain C, Beauchant M. Octreotide in the treatment of the hepatorenal syndrome in cirrhotic patients. J Hepatol 1999; 30: 174

66 Jalan R, Sen S, Steiner C, Kapoor D, Alisa A, Williams R. Extracorporeal liver support with molecular adsorbents recirculating system in patients with severe acute alcoholic hepatitis. J Hepatol 2003; 38: 24-31

S- Editor Liu Y E- Editor Lu W 